\title{
Immunoreactivity of Brazilian HIV Isolates with Different V3 Motifs
}

\author{
R Michael Hendry/ ${ }^{+}$, Carl V Hanson, Vera Bongertz", Mariza Morgado*, \\ Alberto Duarte $^{* *}$, Jorge Casseb ${ }^{* *}$, Luis Brigido ${ }^{* * *}$, Esther Sabino ${ }^{* * *}$, \\ Ricardo Diaz $^{* * * *}$, Bernardo Galvão-Castro**
}

\begin{abstract}
Viral and Rickettsial Disease Laboratory, California Department of Health Services, 2151 Berkeley Way, Berkeley, CA 94704, USA *Departamento de Immunologia, Instituto Oswaldo Cruz, Rio de Janeiro, RJ, Brasil **Laboratório de Immunogenética e Transplante Experimental, Faculdade de Medicina da Universidade de São

Paulo ***Laboratório de Retrovirus, Serviço de Virologia, Instituto Adolfo Lutz, São Paulo, SP, Brasil ****DIPA, Escola Paulista de Medicina, São Paulo, SP, Brasil *****Laboratório Avançado de Saúde Pública, Centro de Pesquisa Gonçalo Moniz-FIOCRUZ, Salvador, BA, Brasil
\end{abstract}

Key words: anti-V3 peptide - neutralization - HIV-1 - antibodies

A successful HIV vaccine must elicit immune responses which recognize variants of HIV circulating in the vaccine target population. It is therefore important to understand HIV genetic and antigenic variation in geographic areas where vaccine trials are anticipated.

In 1991, Carrow et al. published the observation that, like American and African sera, Brazilian $\mathrm{HIV}^{+}$sera reacted extensively with the $\mathrm{V} 3$ region of the MN strain of HIV. These Brazilian sera also readily neutralized HIV-MN (EW Carrow et al. 1991 AIDS Res Human Retrovir 7: 831-837). In 1993, Potts et al. reported sequence heterogeneity in the $\mathrm{V} 3$ region of Brazilian isolates. Based on overall V3 sequences, the majority of Brazilian isolates were more similar to those of North America and Haiti than to African isolates. However, only $59 \%$ of isolates contained proline in the tip of the V3 loop. In this small study, $40 \%$ contained a GPGR motif while $36 \%$ contained a GWGR motif - previously reported in some Japanese isolates (KE Potts et al. 1993 AIDS 7: 11911197).

In 1994, Morgado et al. studied isolates from Rio de Janeiro and São Paulo, and identified one subtype $\mathrm{F}$ isolate and a putative $\mathrm{B} / \mathrm{F}$ recombinant. In this study, octameric sequences at the tip of the Brazilian subtype B sequences were variable and distinct from those in North America and Europe. The GPGR motif was found in only $28.5 \%$ of the isolates, and GWGR in 43\% (M Morgado et al. 1994 AIDS Res Human Retrovir 10: 569-576). Divergence throughout the V3 region suggested that Brazilian isolates might also differ antigeni-

\footnotetext{
${ }^{+}$Corresponding author. Fax: 510-540.3305

Received 7 December 1995

Accepted 10 January 1996
}

cally from prototype subtype B strains (upon which most experimental HIV vaccines are being based).

Sequence analysis, and to a lesser extent heteroduplex mobility analysis, are labor-intensive, and, in any case, possible only when viral isolates or patient PBMCs are available. We therefore undertook a study of antibody binding to defined V3 peptides, using Brazilian sera including many from patients from whom no sequenced isolates were available.

Sequences of the four peptides used in this study are compared with that of the B subtype consensus sequence in Fig. BR1 differs from the subtype B consensus only by substitution of " $W$ " in place of "P" in the GPGR motif. BR2 is a consensus of a group of Brazilian isolates which possess the GWGR motif. Overall, two of the peptides included "P" in the V3 tip motif and two included "W". The peptides were biotinylated at their amino terminus and contained a four amino acid spacer. The biotinylated peptides were captured onto avidin coated microtiter plates.

Sequence of the synthetic peptides used.

Sera were obtained initially from 21 Brazilian patients whose HIV isolates were of known V3 sequence. Binding of these sera to the peptides was determined colorimetrically using an enzymelabeled anti-human-immunoglobulin and a chromogenic substrate. Binding was expressed as the reciprocal of the highest antibody dilution giving an optical density of 0.5 . Serum specimens corresponding to the GWGR motif had highest titers against the GWGR-containing BR-1 and BR-2 
peptides. Specimens corresponding to isolates with "P" in the motif, had titers that were more nearly equal against most of the peptides.

Four different ratios of binding titers (BR1-toSF2, BR1-to-MN, BR2-to-SF2 and BR2-to-MN) were computed. The sum of these four ratios would thus be expected to be high for reactivity with the "W" motif and low for reactivity with the "P" motif. By inspection of the data, we found that ratio-sums above 16 corresponded $100 \%$ with "W" reactivity and ratio-sums below 8 corresponded 100\% with "P" reactivity. Ratios between 8 and 16 were considered indeterminate. Of five specimens with unusual non-W/non-P motifs, two typed by this scheme as "W-like", one as "P-like" and one as indeterminate.

As shown in Table, the ratio-sum technique was applied to sera from Rio de Janeiro, Bahia and São Paulo from patients with no sequenced isolates available. Overall, there were equal numbers of GWGR and GPGR types. While not statistically significant, GPGR predominated in the northernmost region (Bahia), GWGR predominated in the southernmost area (São Paulo), and there were equal numbers in Rio de Janeiro, which lies geographically between the other two sites. Data for São Paulo are shown for equal numbers of samples collected recently and 10 years earlier. Since there is no significant difference between these, it suggests that GWGR was already common in Brazil early in the epidemic. This early, uncommon

\section{TABLE}

Frequency of serologic reactivity to V3 synthetic peptides in Brazilian sera

\begin{tabular}{lccccc}
\hline & & \multicolumn{4}{c}{ Number (\%) of sera } \\
\cline { 3 - 6 } Location & Date & GWGR & GPGR & Indeterminate & Total \\
\hline Bahia & $1988-90$ & $11(27)$ & $27(66)$ & $3(7)$ & 41 \\
Rio & $1988-92$ & $39(48)$ & $37(46)$ & $5(6)$ & 81 \\
São Paulo & 1983 & $18(64)$ & $10(36)$ & 0 & 28 \\
São Paulo & $1993-94$ & $15(54)$ & $13(46)$ & 0 & 28 \\
\hline Total & & $83(47)$ & $87(49)$ & $8(4)$ & 178 \\
\hline
\end{tabular}

GWGR strain may thus be a phylogenetic ancestor of current GWGR strains.

Geometric mean averages of binding titers against each of the six peptides were computed separately for all P-type and for all W-type antisera. In the case of peptides BR2, SF2 and MN, titer differences were especially statistically significant. It is interesting that titers of P-type specimens were especially high against the consensus F-type peptide, in contrast to those of W-type peptides. This is consistent with the "promiscuous", or relatively broad, reactivity of P-type antisera.

These observations suggest that the choice of a GPGR motif strain, such as MN, as the basis for a vaccine may be fortuitous - even in an area such as Brazil where multiple HIV subtypes and V3motif variants coexist. In the context of vaccine development, however, the important issue is the ability of an HIV antigen to elicit broad functional activities such as virus neutralization or HIV-specific cytotoxic T-lymphocytes. Neutralizations of a Brazilian motif-W virus and a Brazilan motif-P were therefore studied. For type-W sera, there was a strong selectivity for neutralization of type-W virus, consistent with the antibody-binding data. In contrast, neutralization of these same viruses by type-P antisera overall occurred without preference for either virus (data not shown).

In conclusion, (1) in Brazil there are subtype$B$ viruses with different V3 motifs; (2) there are two antigenically distinct strains of HIV-1 subtype B cocirculating widely in Brazil; (3) antibody-peptide binding experiments can categorize patients exposed to HIV variants with different V3 motifs, even in the absence of sequenced viral isolates; (4) functional antibody activities, such as virus neutralization, may be correlated with exposure to different V3 motifs; (5) V3-motif analysis and peptide-binding studies may be relevant to the design of HIV vaccines. For example, the GPGR motif of HIV-MN may contribute to its eliciting a more broadly cross-reactive response than would be the case with subtype B viruses having other V3 motifs. 